\title{
Comportamento da Curva de Lactação de Cabras Mestiças Saanen em Função da Suplementação de Concentrado e do Sistema de Produção ${ }^{1}$
}

\author{
Vicente de Paulo Macedo², Júlio Cesar Damasceno ${ }^{3}$, Geraldo Tadeu dos Santos ${ }^{3}$, \\ Elias Nunes Martins ${ }^{3}$, Francisco de Assis Fonseca de Macedo ${ }^{3}$
}

RESUMO - Estudaram-se o comportamento da curva de lactação em 31 cabras mestiças Saanen e o efeito de duas estratégias de suplementação de concentrado: $30 \%$ das exigências em energia líquida da $3^{\mathrm{a}}$ semana até o final da lactação (ESC.1); 60\% das exigências em energia líquida diária da $3^{\mathrm{a}}$ à $13^{\mathrm{a}}$ semana, e $15 \%$ da $14^{\mathrm{a}}$ semana até o final da lactação (ESC.2), em sistemas de produção semiconfinamento e pastagem. Os seguintes modelos foram avaliados: Wood linear e não-linear, Morant linear e não-linear, D-set e Grossman. O modelo Wood não linear foi o que melhor descreveu o comportamento da curva de lactação. A resposta da estratégia de suplementação com concentrado variou em função do sistema de produção. A estratégia de suplementação com concentrado afetou mais intensamente o comportamento da curva de lactação no sistema de produção semi-confinamento. A estratégia ESC.2, de suplementação com concentrado, resultou em mais dias para atingir o pico de produção, no sistema semi-confinamento, e maior produção no pico em ambos sistemas.

Palavras-chave: cabra, curva de lactação, estratégias de suplementação, produção de leite

\section{Lactation Curve Behavior of Cross Breed Saanen Dairy Goats in Function of the Concentrate Supplementation and the Production System}

\begin{abstract}
The behavior of lactation curve of 31 crossbred Saanen dairy goats and the effect of two strategies of supplementation with concentrate: $30 \%$ of requirements in net energy from third week until the end of lactation (ESC.1); or $60 \%$ of requirements in daily net energy from the $3^{\text {rd }}$ to the $13^{\text {th }}$ week and $15 \%$ from $14^{\text {th }}$ until the end of lactation (ESC.2), in a pasture or semi-confined systems, were studied. The following models were evaluated: Wood linear and non-linear, Morant linear and non-linear, D-set and Grossman. Nonlinear Wood model was the best in describing the behavior of lactation curve. The responses to the strategy of supplementation with concentrate were different in function of production systems. The strategy of supplementation affected the behavior of lactation curve more intensely in the semi-confined system. The ESC.2 strategy of supplementation resulted in more days to reach the production pick, in semi-confined system, and larger production in the lactation pick in both systems.
\end{abstract}

Key Words: goat, lactation curve, milk production, strategies of supplementation

\section{Introdução}

Curvas de lactação representam a relação entre produção de leite e o tempo após o parto. O conhecimento do comportamento da curva de lactação é de suma importância para facilitar o manejo nutricional de animais em lactação, pois possibilita a estimativa da produção total de leite, do pico de produção e da persistência de produção.

Diferentes modelos que descrevem o comportamento da curva de lactação são apresentados na literatura, contudo, os parâmetros utilizados nos modelos, às vezes, não se ajustam adequadamente. Modelos mecanicistas, baseados na biologia da lactação, têm sido propostos por NEAL e THORNLEY (1983), mas esses são muito complexos para aplicação em rotinas fora de pesquisa. GIPSON (1989) relata que modelos empíricos têm sido utilizados para simulação da lactação e para previsões de futuras produções.

GROSSMAN e KOOPS (1988) propõem a utilização de modelo com seis parâmetros de funções difásicas, dividindo a lactação em duas fases: uma, representando o pico de produção e a outra, a persistência da lactação. O uso de seis parâmetros torna o modelo mais flexível, comparado a outros modelos, e podem ser interpretados biologicamente (GIPSON e GROSSMAN, 1989). Modelos com quatro

${ }^{1}$ Parte da dissertação do primeiro autor apresentada ao PPZIDZOIUEM, Maringá, como um dos requisitos para a obtenção do título de Mestre em Produção Animal.

2 Zootecnista, aluno do curso de pós-graduação em Zootecnia - UNESP - Botucatu.

${ }^{3}$ Professores do Departamento de Zootecnia da UEM, Av. Colombo, 5790 - 87020-900, Maringá - PR. E.mail: jcdamasceno@uem.br 
parâmetros, são sugeridos por MORANT e GNANASAKTHY (1990), nos quais, há menores correlações entre os parâmetros, o que possibilita interpretação independentemente um dos outros.

Existem poucos trabalhos com o objetivo de ajustar modelos para cabras lactantes, embora alguns destes tenham sido realizados (DUDOUET, 1982). De acordo com GIPSON (1989), e GIPSON e GROSSMAN (1989), o modelo de Grossman foi eficiente, para descrever o comportamento da curva de lactação.

DAMASCENO et al. (1997) obtiveram aumentos lineares na produção de leite, com incrementos na suplementação de 15 até $60 \%$ das exigências diárias em energia para cabras lactantes. Neste estudo, os melhores resultados coincidiram com a maior produção no pico, associada à maior duração da lactação. A suplementação de $30 \%$ das exigências diárias dos animais em energia líquida supridas pelo concentrado ao longo da lactação, propiciou os melhores retornos econômicos.

Pesquisas, avaliando estratégias de fornecimento de concentrados na lactação de cabras lactantes, em diferentes sistemas de produção, são escassas na literatura. Por esta razão, realizou-se este trabalho com os objetivos de definir o modelo que melhor descreve o comportamento da curva de lactação de cabras mestiças Saanen e avaliar o efeito de duas estratégias de suplementação com concentrado, em sistema de produção em pastagem e em semi-confinamento.

\section{Material e Métodos}

O experimento foi conduzido no setor de caprinocultura da Fazenda Experimental de Iguatemi (FEI), Universidade Estadual de Maringá, no período de agosto de 1997 a outubro de 1998. Foram utilizadas 31 cabras em lactação, com grau de sangue $3 / 4,7 / 8$ e 15/16 da raça Saanen, e ordem de lactação $1^{\mathrm{a}}, 2^{\mathrm{a}}, 3^{\mathrm{a}}$ e $4^{\mathrm{a}}$. Para a formação dos lotes, foram levados em consideração: ordem de lactação e grau de sangue.

O setor conta com um aprisco, dividido em boxes individuais, contendo bebedouros, cochos e canzis para alimentação dos animais, sala de ordenha e uma área de pastagem de estrela africana (Cynodon nlemfuensis).

Os valores médios, máximos e mínimos para produção de leite e duração do período de lactação encontram-se na Tabela 1.

Os tratamentos foram duas estratégias de

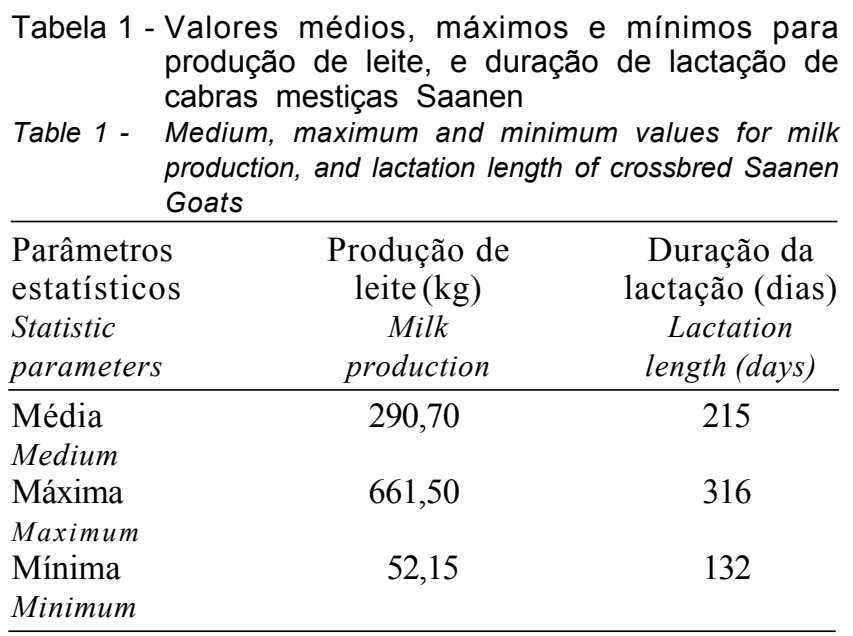

suplementação com concentrado e dois sistemas de produção.

Foram testadas as seguintes estratégias de suplementação com concentrados (ESC): $30 \%$ das exigências em energia líquida (NRC 1981) da $3^{\mathrm{a}}$ semana até o final da lactação (ESC.1); 60\% das exigências em energia líquida diária da $3^{\mathrm{a}}$ à $13^{\mathrm{a}}$ semana, e $15 \%$ (NRC 1981) da $14^{\text {a }}$ semana até o final da lactação (ESC.2). A quantidade de concentrado fornecida foi corrigida, semanalmente, de acordo com a produção de leite e peso vivo dos animais. Estas estratégias de suplementação com concentrado foram avaliadas em dois sistemas de produção: semi-confinamento (Sis. 1) e em pastagem (Sis.2).

As Tabelas 2 e 3 apresentam as composições químicas do concentrado, pasto e feno utilizados ao longo do período experimental. Os concentrados fornecidos aos animais em crescimento e animais adultos continham $72 \%$ de milho e $28 \%$ de farelo de soja, $75 \%$ de milho e $25 \%$ de farelo de soja, respectivamente.

Foi considerado animal em crescimento, aquele de primeira ou segunda lactação. Para o cálculo das exigências nutricionais, consideraram-se, além da produção de leite e peso vivo dos animais, taxas de ganho de peso, que deveriam ser realizadas para que os animais de $1^{\mathrm{a}} \mathrm{e} 2^{\mathrm{a}}$ parição atingissem o peso adulto na idade adequada (em torno de 700 dias).

Os animais eram ordenhados pela manhã $(6 \mathrm{~h})$ e à tarde (15h e30 min.). Após a ordenha da manhã, eram levados ao pasto e, após a ordenha da tarde, os animais do sistema semi-confinamento eram recolhidos ao galpão, onde permaneciam durante a noite e recebiam feno de gramíneas do gênero Cynodon à

Rev. bras. zootec., 30(6S):2093-2098, 2001 
Tabela 2 - Composição química e digestibilidade In Vitro da matéria seca dos concentrados

Table 2 - Chemical composition and in vitro dry matter digestibility of concentrates

\begin{tabular}{lcc}
\hline $\begin{array}{l}\text { Variáveis }(\% \text { da MS) } \\
\text { Variables }(\% \text { of } D M)\end{array}$ & $\begin{array}{c}\text { Cabras } \\
\text { adultas } \\
\text { Adult goats }\end{array}$ & $\begin{array}{c}\text { Cabras } \\
\text { jovens } \\
\text { Young goats }\end{array}$ \\
\hline PB & 21,37 & 21,70 \\
$C P$ & 14,68 & 14,30 \\
FDN & & \\
$N D F$ & 5,06 & 4,95 \\
FDA & & \\
ADF & 89,59 & 87,30 \\
DIVMS & &
\end{tabular}

${ }^{1} \mathrm{~PB}$ : proteína bruta; FDN: fibra em detergente neutro; FDA: fibra em detergente ácido; DIVMS: digestibilidade in vitro da matéria seca.

${ }^{1} \mathrm{CP}$ : crude protein; NDF: neutral detergent fiber; ADF: acid detergent fiber; IVDMD: in vitro dry matter digestibility.

vontade (@25\% de sobras). O feno disponível aos animais do sistema de produção semi-confinamento, sofreu um processo de moagem, transformando-se em alimento com partículas finas e pulverulento. Os animais do sistema de produção em pastagem permaneceram durante todo o tempo no pasto, tendo como única fonte de volumoso a pastagem. Água e sal mineral foram fornecidos à vontade. Cada animal recebeu concentrado, na quantidade determinada, após ordenha da manhã e tarde.

A produção de leite $(\mathrm{kg} / \mathrm{dia})$ foi o resultado de duas ordenhas diárias, obtidas semanalmente. A variação do peso e a condição corporal ao longo do período experimental foram acompanhadas mensalmente, mediante pesagem e avaliação, através de palpação dos animais. A $1^{\text {a }}$ pesagem foi realizada logo após o parto. A metodologia para avaliação corporal dos animais se baseia na palpação em duas regiões, uma ao nível dorso lombar (apófises) e outra no externo, sendo que o estado corporal do animal é a média das duas notas (de 0 a 5 ), coletadas ao nível dorso lombar e no externo, em uma escala de 0,25.

A disponibilidade de Matéria Seca (MS) da pastagem (kg MS/ha), ao longo do experimento, foi determinada a intervalos de 15 dias, através do método da dupla-amostragem (WILM et al., 1944).

A pastagem foi manejada com o objetivo de manter um resíduo disponível de, aproximadamente, $3.000 \mathrm{~kg}$ de $\mathrm{MS} / \mathrm{ha}$ ao longo de todo o período experimental. Isto foi obtido através de calagem $(\mathrm{V}=$ $60 \%)$ e adubação adequada $\left(137,13 \mathrm{~kg} \mathrm{~N}, 80 \mathrm{~kg} \mathrm{P}_{2} \mathrm{O}_{5}\right.$ e $100 \mathrm{~K}_{2} \mathrm{O} /$ ha) e pelo ajuste da carga animal.

Foram realizadas vacinações contra febre aftosa duas vezes ao ano, em maio e outubro. A cada 30 dias, os cascos dos animais foram inspecionados.

As aplicações de antihelmínticos foram feitas, apenas, nos animais que apresentavam 1.000 ou mais ovos por grama de fezes, e com intervalo de aplicações de, no mínimo, 45 dias.

Foram realizadas amostragens mensais do feno e dos concentrados, determinando-se fibra em detergente neutro (FDN), fibra em detergente ácido (FDA), nitrogênio insolúvel em detergente ácido (NIDA), proteína bruta $(\mathrm{PB})$ e digestibilidade in vitro da

Tabela 3 - Valores médios, máximos e mínimos da composição química e da digestibilidade in vitro da matéria seca (DIVMS) do pasto e do feno

Table 3 - Medium, maximum and minimun values of the chemical composition and in vitro dry matter digestibility (IVDMD) of grass and hay

\begin{tabular}{|c|c|c|c|c|c|c|}
\hline \multirow[t]{2}{*}{$\begin{array}{l}\text { Variáveis }(\% \text { da MS })^{1} \\
\text { Variables }(\% D M)\end{array}$} & \multicolumn{3}{|c|}{$\begin{array}{l}\text { Pasto } \\
\text { Grass }\end{array}$} & \multicolumn{3}{|c|}{$\begin{array}{l}\text { Feno } \\
\text { Hay }\end{array}$} \\
\hline & $\begin{array}{l}\text { Média } \\
\text { Medium }\end{array}$ & $\begin{array}{l}\text { Máximo } \\
\text { Maximum }\end{array}$ & $\begin{array}{l}\text { Mínimo } \\
\text { Minimum }\end{array}$ & $\begin{array}{l}\text { Média } \\
\text { Medium }\end{array}$ & $\begin{array}{l}\text { Máximo } \\
\text { Maximum }\end{array}$ & $\begin{array}{l}\text { Mínimo } \\
\text { Minimum }\end{array}$ \\
\hline $\begin{array}{l}\mathrm{PB} \\
C P\end{array}$ & 9,60 & 11,43 & 7,13 & 6,75 & 10,02 & 4,13 \\
\hline $\begin{array}{l}\text { FDN } \\
N D F\end{array}$ & 77,56 & 80,42 & 74,55 & 81,30 & 85,39 & 74,96 \\
\hline $\begin{array}{l}\text { FDA } \\
A D F\end{array}$ & 41,01 & 43,96 & 37,02 & 44,21 & 48,13 & 40,70 \\
\hline $\begin{array}{l}\text { NIDA } \\
A D I N\end{array}$ & 2,29 & 2,73 & 1,82 & 1,96 & 2,91 & 1,02 \\
\hline DIVMS & 50,56 & 55,16 & 43,83 & 45,28 & 53,61 & 31,90 \\
\hline
\end{tabular}

IVDM

${ }_{1}$ PB: proteína bruta; FDN: fibra em detergente neutro; FDA: fibra em detergente ácido; NIDA: nitrogênio insolúvel em detergente ácido; DIVMS: digestibilidade in vitro da matéria seca.

${ }^{1}$ CP: crude protein; NDF: neutral detergent fiber; ADF: acid detergent fiber; ADIN: acid detergent insoluble nitrogen; IVDMD:

Rev. bras. zootec., 30(6S):2093-2098, 2001 
matéria seca (DIVMS). As amostras foram secas em estufa de ar forçado a $55^{\circ} \mathrm{C}$ por $72 \mathrm{~h}$ e, posteriormente, moídas em moinho de faca, em peneira de crivos com diâmetro de $1 \mathrm{~mm}$. Nestas amostras foram realizadas análises de MS, PB e NIDA, segundo SILVA (1990), e de FDA e FDN, segundo GOERING e VAN SOEST (1970), utilizando-se digestor de fibras Fiber Analyser (ANKOM Co. -NY-USA).

A digestibilidade in vitro da MS foi feita de acordo com técnica descrita por TILLEY e TERRY (1963), adaptada para o uso do rúmen artificial (ANKOM Co. -NY-USA), conforme descrito por GARMAN et. al. (1997). As análises laboratoriais foram realizadas no Laboratório de Análises de Alimento e Nutrição e no Laboratório de Digestibilidade e Metabolismo Animal do Departamento de Zootecnia da Universidade Estadual de Maringá.

Para descrever o comportamento da curva de lactação dos animais, seis modelos foram estudados: Wood linear $\log (\mathrm{y})=\mathrm{A}+\mathrm{B} \log (\mathrm{n})$ - Cn; Wood nãolinear $\mathrm{y}=\mathrm{An}^{\mathrm{B}} \exp (-\mathrm{Cn})$; Morant linear $\log (\mathrm{y})=\mathrm{A}+$ $\mathrm{Bn}$ ' $+\mathrm{Cn}$ ' $+\mathrm{D} / \mathrm{n}$; Morant não-linear y $=\mathrm{A} \exp \left(\mathrm{Bn}^{\prime}+\right.$ $\mathrm{Cn}^{\prime 2}+\mathrm{D} / \mathrm{n} ; \mathrm{D}-\mathrm{set} \mathrm{y}=\mathrm{A} \exp \left(\mathrm{B}\left(1+\mathrm{n}^{\prime} / 2\right) \mathrm{n}^{\prime}+\mathrm{Cn}^{\prime 2}-\right.$ $1,01 / \mathrm{n})$ e Grossman $\mathrm{y}=\mathrm{A}_{1} \mathrm{~B}_{1}\left\{1-\tanh ^{2}\left[\mathrm{~B}_{1}\left(\mathrm{n}-\mathrm{C}_{1}\right)\right]\right\}$ $+\mathrm{A}_{2} \mathrm{~B}_{2}\left\{1-\tanh ^{2}\left[\mathrm{~B}_{2}\left(\mathrm{n}-\mathrm{C}_{2}\right)\right]\right\}$.

Animais que não tiveram a curva de lactação ajustada, apropriadamente, foram retirados das análises, perfazendo um total de 4 animais, sendo 1 animal de cada tratamento. Esses animais tiveram um período de lactação e produção total de leite em torno de 133 dias e $53 \mathrm{~kg}$ de leite, respectivamente.

Utilizou-se como critérios para a escolha do melhor modelo, o comportamento dos resíduos (real - estimado) ao longo da lactação; a variância residual e a habilidade dos parâmetros em descrever os efeitos biológicos. Os modelos foram ajustados para cada animal e, em seguida, os valores dos resíduos eram obtidos por diferença entre a produção real e a estimada.

Os efeitos dos tratamentos nos parâmetros do modelo escolhido foram avaliados através de análise de variância de acordo com o modelo estatístico a seguir:

$$
\begin{aligned}
\mathrm{Y}_{\mathrm{ijklmn}}= & \mu+\mathrm{C}_{\mathrm{i}}+\mathrm{S}_{\mathrm{j}}+\mathrm{CS}_{\mathrm{ij}}+\mathrm{O}_{\mathrm{k}}+\mathrm{G}_{1}+\mathrm{E}_{\mathrm{m}}+\mathrm{b}_{1} \\
& (\mathrm{~L}-\mathrm{LM})+\mathrm{b}_{2}(\mathrm{P}-\mathrm{PM})+\varepsilon_{\mathrm{ijklmn}}
\end{aligned}
$$

em que: $Y_{\text {ijklmn }}=$ observação no animal n da estratégia de suplementação com concentrado $i$, do sistema de produção $\mathrm{j}$, ordem de parto $\mathrm{k}$, grau de sangue 1 , e época de parto $\mathrm{m} ; \mu=$ constante geral; $\mathrm{C}_{\mathrm{i}}=$ efeito da suplementação com concentrado $i$; $(i=1$ e 2$)$; $\mathrm{S}_{\mathrm{j}}=$ efeito do sistema de produção $\mathrm{j} ;(\mathrm{j}=1$ e 2$)$;
$\mathrm{CS}_{\mathrm{ij}}=$ efeito da interação entre a suplementação com concentrado i e o sistema de produção $\mathrm{j} ; \mathrm{O}_{\mathrm{k}}=$ efeito da ordem de lactação $\mathrm{k} ;(\mathrm{k}=1 \ldots 4) ; \mathrm{G}_{1}=$ efeito do grau de sangue $1 ;(1=1 \ldots 3) ; \mathrm{E}_{\mathrm{m}}=$ efeito da época de parto $m ;(m=1$ e 2$) ; b_{1}=$ coeficiente linear de regressão da variável $\mathrm{Y}$ em função da produção de leite nos primeiros 21 dias pós-parto; $b_{2}=$ coeficiente linear de regressão da variável Y em função do peso ao parto; $\mathrm{L}=$ produção de leite do animal 1 nos 21 dias pós-parto; $\mathrm{LM}=$ média da produção de leite nos 21 dias pós-parto; $\mathrm{P}=$ peso ao parto do animal 1 ; $\mathrm{PM}=$ média de peso ao parto; $\varepsilon_{\mathrm{ijklmn}}=$ erro aleatório associado a cada observação.

\section{Resultados e Discussão}

O coeficiente de determinação $\left(\mathrm{R}^{2}\right)$ e a variância residual dos modelos utilizados encontram-se na Tabela 4, cujos resultados indicam que os menores valores de variância residuais foram obtidos para os modelos Wood não-linear e Morant linear, e os maiores para Morant não-linear e D-set. De maneira geral, os coeficientes de determinação dos modelos estudados foram altos, exceto para os modelos Wood linear e Morant linear.

Na Figura 1 observa-se que todos os modelos estimaram adequadamente as produções na porção intermediária da curva de lactação, mas a habilidade dos modelos estimarem as produções, no início e no final da curva, foi distinta entre os mesmos.

Tabela 4 - Coeficiente de determinação e variância residual dos modelos

Table 4 - Standard deviation and variance of residual of the models

\begin{tabular}{lcc}
\hline $\begin{array}{l}\text { Modelos } \\
\text { Models }\end{array}$ & $\begin{array}{c}\text { Coeficiente de } \\
\text { determinação }\left(\mathrm{R}^{2}\right) \\
\text { Coefficient of } \\
\text { determination }\left(R^{2}\right)\end{array}$ & $\begin{array}{c}\text { Variância } \\
\text { Variance }\end{array}$ \\
\hline $\begin{array}{l}\text { Wood linear } \\
\begin{array}{l}\text { Wood linear } \\
\text { Wood não-linear }\end{array}\end{array}$ & 0,47 & 0,2949 \\
$\begin{array}{l}\text { Wood non-linear } \\
\text { Morant linear }\end{array}$ & 0,84 & 0,0530 \\
$\begin{array}{l}\text { Morant linear } \\
\text { Morant não-linear }\end{array}$ & 0,48 & 0,0540 \\
$\begin{array}{l}\text { Morant non-linear } \\
\text { D-set }\end{array}$ & 0,84 & 2,1700 \\
$\begin{array}{l}\text { D-set } \\
\text { Grossman } \\
\text { Grossman }\end{array}$ & 0,84 & 0,4500 \\
\hline
\end{tabular}

Rev. bras. zootec., 30(6S):2093-2098, 2001 


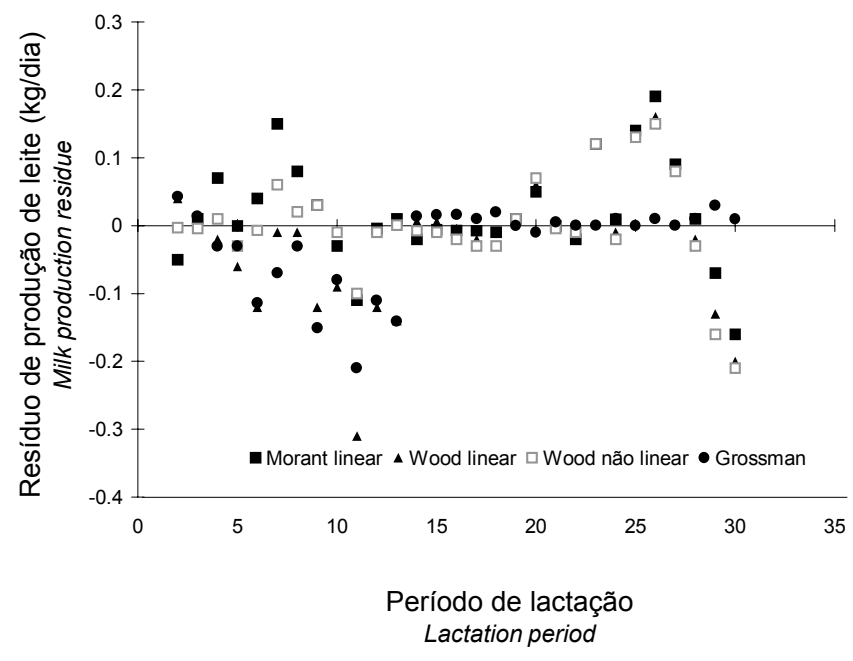

Figura 1 - Comportamento residual de produção de leite ao longo da lactação.

Figure 1 - Residual behavior of milk production during lactation period.

Os modelos D-set e Morant não-linear não foram mostrados no gráfico, uma vez que exibiram altos valores de variância residual e as estimativas diferiram consideravelmente dos valores observados, principalmente, no início e final da lactação.

Os modelos Morant linear e Grossman apresentaram baixos valores residuais no final da curva de lactação mas foram variáveis na porção inicial da curva. Embora o modelo Wood não-linear tenha apresentado maior variação residual na porção final da curva de lactação comparado ao Morant linear e Grossman, as estimativas, no início da lactação, foram as melhores.

Associando-se a menor variância residual ao comportamento mais adequado das estimativas ao longo da lactação, concluiu-se que o modelo Wood não-linear foi o que melhor descreveu o comportamento da curva de lactação das cabras utilizadas no presente experimento.

KELLOGG et. al. (1977), BASANT e BLAT (1978), MADALENA et. al. (1979) e WOOD (1980) relatam que o modelo de Wood não-linear tem demonstrado ser o que melhor representa o comportamento da curva de lactação. Em estudos com cabras, GIPSON e GROSSMAN (1989), WILLIAMS (1993) observaram que as diferenças entre resíduos do modelo Wood não-linear comparado a modelos contendo mais parâmetros foram relativamente menores, sugerindo que este modelo pode ser adequado para estudar os fatores que afetam o comportamento da curva de lactação em cabras, concordando com o observado no presente trabalho.

A partir dos parâmetros do modelo, foram derivadas as variáveis dia de produção no pico (P, em dias) e produção no pico ( $\mathrm{PP}, \mathrm{kg} / \mathrm{dia})$, calculadas da seguinte forma: $\mathrm{P}=\mathrm{b} / \mathrm{c} ; \mathrm{PP}=\mathrm{a}(\mathrm{b} / \mathrm{c})^{\mathrm{b}} \mathrm{e}^{-\mathrm{b}}$.

Na Tabela 5 encontram-se os valores médios dos parâmetros do modelo Wood não-linear, bem como das variáveis derivadas destes parâmetros. Verifica-se que a resposta dos parâmetros à estratégia de suplementação com concentrado (ESC) variou $(\mathrm{P}<0,05)$, em função do sistema de produção.

No sistema de produção semi-confinamento, a

Tabela 5 - Valores médios dos parâmetros do modelo Wood não linear, em dois sistemas e em duas estratégias de suplementação com concentrado

Table 5 - Average values of Wood non linear model, in two production system and two supplementation strategies with concentrate

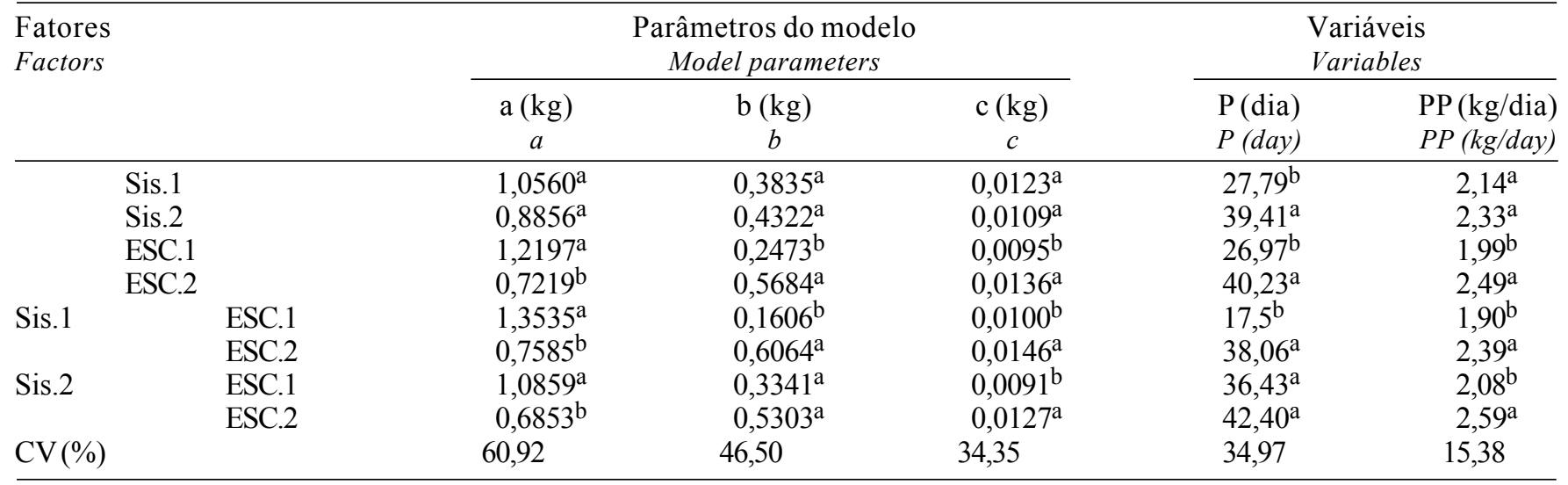

$a, b=$ Médias com letras distintas na mesma coluna diferem $(P \leq 0,05)$.

$a, b=$ Average with different letters at the same row are different $(P \leq .05)$.

Rev. bras. zootec., 30(6S):2093-2098, 2001 
ESC.2 (60\% das exigências em energia líquida diária da $3^{\mathrm{a}}$ à $13^{\mathrm{a}}$ semana, e $15 \%$ da $14^{\mathrm{a}}$ semana, até o final da lactação) resultou em maiores valores para $b$ (taxa de acréscimo de produção até o pico), $c$ (taxa de declínio de produção após o pico) $\mathrm{P}$ (dia de produção no pico) e PP (produção no pico), mas inferior para $a$ (produção inicial). Já, no sistema de produção em pastagem, apenas os parâmetros $a, c$ e $\mathrm{PP}$ sofreram efeito $(\mathrm{P}<0,05)$ da estratégia de suplementação com concentrado; em que a ESC.2 resultou em maiores valores de $c$ e PP, e inferior de $a$. Estes resultados indicam que maiores aportes de concentrado no início da lactação e redução, após algumas semanas do pico, baseado nas exigências diárias dos animais, resultam em benefícios ao animal, permitindo-o alcançar maior produção no pico de lactação.

\section{Conclusões}

O modelo de Wood não-linear foi o que melhor descreveu o comportamento da curva de lactação de cabras mestiças Saanen.

O efeito da estratégia de suplementação com concentrado, na curva de lactação, depende do sistema de produção.

A estratégia de suplementação com concentrado afeta mais intensamente o comportamento da curva de lactação no sistema de produção semiconfinamento.

A estratégia de suplementação com concentrado ESC.2 (60\% das exigências em energia líquida diária da $3^{\mathrm{a}}$ à $13^{\mathrm{a}}$ semana, e $15 \%$ da $14^{\mathrm{a}}$ semana até o final da lactação) resulta em mais dias para atingir o pico de produção, no sistema semi-confinamento, e maior produção no pico em ambos sistemas.

\section{Referências Bibliográficas}

BASANT, S., BLAT, P.N. 1978. Models of lactation of curves for Hariana cattle. J. Anim. Sci., 48:791-796.

DAMASCENO, J.C., FERREIRA, A.C.D., GEAHL, S.E. et al. 1997. Responses of the dairy goats supplemented with different levels of concentrate. J. Dairy Sci., 80(supl.1):462.
DUDOUET, E. 1982. Theoretical lactation curve of the goat and its applications. Le Point Vet., 14(68):53-61.

GARMAN, C.L., HOLDEN, L.A., KANE, H.A. 1997. Comparison of in vitro dry matter digestibility of nine feedstuffs using three methods of analysis. J. Dairy Sci., 80 (suppl. 1):260.

GIPSON, T.A. Lactation curves in dairy goats. (Ph.D. Thesis), University of Illinois, Urbana, USA. 1989.

GIPSON, T.A., GROSSMAN, M. 1989. Diphasic analysis of lactation curves in dairy goats. J. Dairy Sci., 72:1035-1044.

GOERING, H.K., VAN SOEST, P.J. Forage fiber analyses. USDA Handbook. n. 379, Washington, 1970.

GROSSMAN, M., KOOPS, W.J. 1988. Multiphasic analysis of lactation curves in dairy cattle. J. Dairy Sci., 71:1598-1508.

KElloGG, D.W., URQUHART, S., ORTEGA, A.J. 1977. Estimating Holstein lactation curves with a gamma curve. J. Dairy Sci., 60:1308-1315.

MADALENA, F.E., MARTINEZ, M.L., FREITAS, A.F. 1979. Lactation curves of Holstein-Friesian and Holstein x Gir cows. Anim. Prod., 29:101-107.

MORANT, S.V., GNANASAKTHY, A. 1990. A new approach to the mathematical formulation of lactation curves. Anim. Prod., 49:151-162.

NEAL, H.D., THORNLEY, J.H.M. 1983. The lactation curve in cattle: a mathematical model of the mammary gland. J. Agric. Sci., 101:389-400.

NATIONAL RESEARCH COUNCIL - NRC. 1981. Nutrient requirement of goats. 15.ed. Washington, D.C.: National Academy Press. 91p.

SILVA, D.J. 1990. Análise de alimentos (métodos químicos e biológicos). 2.ed. Viçosa, MG: UFV. 65p.

TILLEY, M.A., TERRY, R.A. 1963. A two-stage technique for the in vitro digestion of forage crops. J. Brit. Grassland Soc., 18:104-111.

WILLIAMS, J.C. 1993. An empirical model for the lactation curve of white British dairy goats. Anim. Prod., 57:91-97.

WILM, H.G., COSTELlO, O.F., KLIPPLE, G.E. 1944. Estimating forage yield by the double sampling method. J. Am. Soc. Agr., 36(1):194-203.

WOOD, P.D.P. 1980. Breed variation in the shape of the lactation curve of cattle and their implications for efficiency. Anim. Prod., 34:133-141.

Recebido em: 30/06/99

Aceito em: 14/05/01 